\title{
SINTONIA DE UM CONTROLADOR PID EM UM SISTEMA DE CONTROLE DE NÍVEL DE TANQUES EM SÉRIE UTILIZANDO UM SOFTWARE GRATUITO
}

\author{
A. M. RIBEIRO ${ }^{1}$ e R. B. SANTOS \\ Instituto Federal de Educação, Ciência e Tecnologia do Sul de Minas \\ ${ }^{1}$ E-mail para contato: alexandremribeiro96@hotmail.com
}

\begin{abstract}
RESUMO - Controladores PID são largamente utilizados no controle de processos químicos. No entanto, muitos dos controladores encontrados nos sistemas químicos são mal sintonizados. Ajustar parâmetros referentes a controladores PID é de extrema importância, pois pode ocasionar perda de material, além de poder oferecer riscos quanto a segurança. No presente trabalho, utilizando a plataforma de simulação $x \cos$ do software livre Scilab, foi implementada a modelagem de um sistema de dois tanques em série, a fim de controlar o nível do segundo tanque através de um controlador PID. Os parâmetros do controlador foram ajustados através dos métodos de sintonia Ziegler-Nichols II e Tyreus-Luyben em malha fechada, comparando os dois resultados e fazendo um ajuste fino em cima do ajuste com menor erro.
\end{abstract}

\section{INTRODUÇÃO}

Todos os sistemas químicos estão sujeitos a perturbações, ou seja, distúrbios no processo. Os processos em malha fechada possuem a vantagem de manter o valor de uma variável controlada o mais próximo do valor de setpoint de maneira automatizada, sem a necessidade da atuação de um operador em campo para este fim, além de possuírem alto índice de confiabilidade garantindo maior segurança ao processo.

Para implementação do controlador, é necessário conhecimento das variáveis de processo e sua resposta a eventuais distúrbios, o que pode ser analisado experimentalmente, porém, pode acarretar um alto risco relacionado a segurança e custo elevado de perdas de matéria primas. Uma alternativa é modelar matematicamente o sistema químico a partir de princípios físicos e químicos, estimando comportamento perante as perturbações com o auxílio de softwares simuladores como fizeram Martins (2005) e Jabuonski et al. (2003).

Para implementação de malha fechada é necessário sintonizar os ganhos $\mathrm{K}_{\mathrm{C}}$ (proporcional), $\mathrm{K}_{\mathrm{I}}$ (integrativo) e $\mathrm{K}_{\mathrm{D}}$ (derivativo) do controlador. As sintonias do controlador PID (proporcional integral e derivativo) neste trabalho foram feitas segundo os métodos de Ziegler-Nichols II e Tyreus-Luybe (Franchi, 2011; Haugen, 2010).

O controle de nível em tanques de processos químicos é necessário devido as possíveis perturbações nas vazões volumétricas de entrada e saída, podendo, por exemplo, transbordar se não estiver em malha fechada. Jabuonski et al. (2003) aborda tal controle com auxílio de simulação computacional, obtendo resultados promissores. 
Devido a importância da sintonia dos controladores, o presente trabalho tem como objetivo a implementação de um controlador PID para controlar o nível em um sistema de tanques em serie, através da utilização do software gratuito Scilab ajustar os parâmetros do controlador através dos métodos de sintonia Ziegler-Nichols II e Tyreus-Luyben, fazendo uma comparação entre os dois métodos e o critério de integração do erro para realizar a sintonia fina do controle.

\section{MATERIAIS E MÉTODOS}

Foi proposto controlar o nível do segundo tanque $\left(\mathrm{h}_{2}\right)$ do sistema representado pela Figura 1, sistema bastante comum na indústria química.

Figura 1 - Tanques em série.

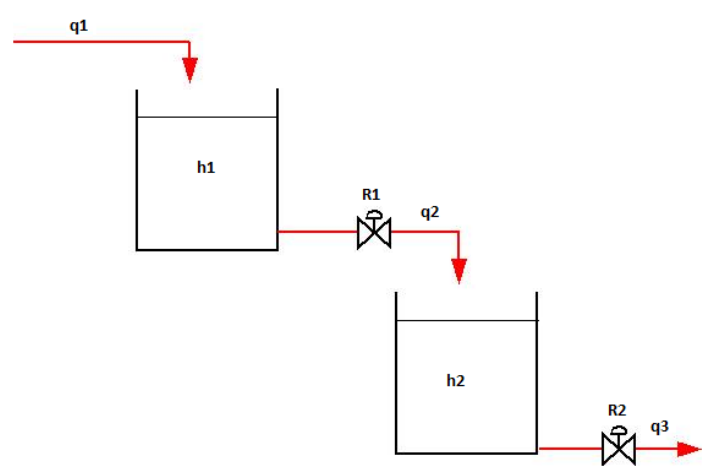

As áreas de seção transversal de ambos os tanques (A) são de $10 \mathrm{ft}^{2}$, as resistências de ambas as válvulas $\left(\mathrm{R}_{1}=\mathrm{R}_{2}\right)$ são de $0,1 \mathrm{ft} /\left(\mathrm{ft}^{3} / \mathrm{min}\right)$, vazão volumétrica de entrada no primeiro tanque $\left(\mathrm{q}_{1}\right)$ de $30 \mathrm{ft}^{3} / \mathrm{min}$, vazão volumétrica de saída do primeiro tanque e entrada do segundo $\left(\mathrm{q}_{2}\right)$ é expressa por $\mathrm{q}_{2}=\mathrm{h}_{1} / \mathrm{R}_{1}$ e vazão volumétrica de saída do segundo tanque $\left(\mathrm{q}_{3}\right)$ expressa por $\mathrm{q}_{3}=\mathrm{h}_{2} / \mathrm{R}_{2}$.

Modelando matematicamente a partir do volume do primeiro tanque obtemos a primeira equação diferencial ordinária (Equação 1).

$\frac{d h_{1}}{d t}=\frac{1}{A}\left(q_{1}-\frac{h_{1}}{R_{1}}\right)$

Para o segundo tanque, tem-se a taxa conforme a Equação 2.

$\frac{d h_{2}}{d t}=\frac{1}{A}\left(\frac{h_{1}}{R_{1}}-\frac{h_{2}}{R_{2}}\right)$

Implementou-se a Equação 1 e a Equação 2 na plataforma xcos do software gratuito Scilab versão 5.5.2, cuja interface com a modelagem é apresentada na Figura 2.

Para obtenção dos parâmetros da função transferência do processo $\left(G_{P}(s)\right)$, utilizou-se os resultados da modelagem do segundo tanque, já que a proposta é de controlar $\mathrm{h}_{2}$ (altura do 
segundo tanque). Para isso, foi provocada uma perturbação degrau na vazão de entrada do primeiro tanque, alterando a vazão de $20 \mathrm{ft}^{3} /$ min para $50 \mathrm{ft}^{3} / \mathrm{min}$.

Figura 2 - Interface do $x \cos$ com a modelagem do processo

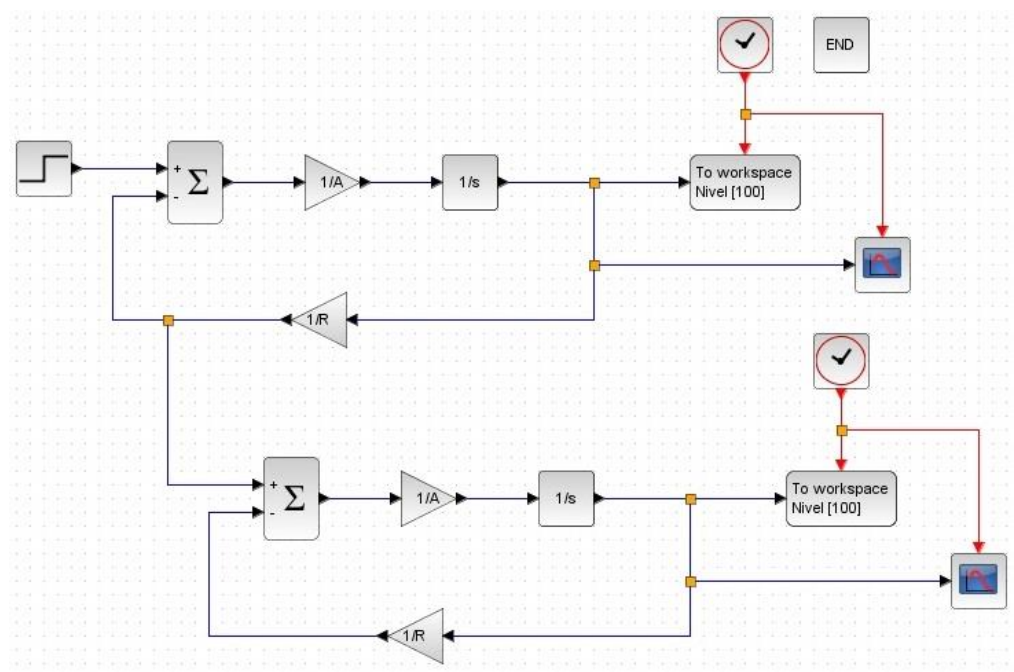

Com a função de transferência do processo foi implementado controlador PID, ajustado os parâmetros com os métodos de sintonia Ziegler-Nichols II e Tyreus-Luyben e através do critério de integração do erro (equação 3) encontrou-se valores que minimizam o erro, sendo então selecionados para utilização no controle.

$\operatorname{ITAE}=\int t \cdot|e(t)| d t$

\section{RESULTADOS E DISCUSSÕES}

\subsection{Obtenção da função transferência (identificação do processo)}

Com a perturbação na vazão de entrada no primeiro tanque, houve uma variação de 30 $\mathrm{ft}^{3} / \mathrm{min}$ (variável de entrada), notou-se que a variação do nível do segundo tanque (variável de saída) até atingir estado estacionário foi de aproximadamente $3 \mathrm{ft}$ (conforme Figura 3), então pode-se determinar o ganho do processo $\left(\mathrm{K}_{\mathrm{P}}\right)$ de 0,1 . Traçou-se uma reta tangente ao ponto de inflexão na curva de reação do processo para obtenção da constante de tempo $(\tau)$ 2,75 min e do tempo morto $\left(t_{D}\right)$ de aproximadamente 0,25 minutos (aproximação da função de transferência de segunda ordem para primeira ordem com tempo morto). A função transferência do processo é apresentada pela Equação 4.

$G_{P}(s)=\frac{0,1 e^{-0,25 s}}{2,75 s+1}$

\subsection{Implementação da malha fechada}

Implementando um sistema de controle PID feedback e assumindo que as funções de transferência do elemento atuador $\mathrm{G}_{\mathrm{F}}(\mathrm{s})$ e do sensor $\mathrm{G}_{\mathrm{M}}(\mathrm{s})$ sejam iguais a 1 , construiu-se o diagrama de controle no simulador conforme a Figura 4. 
Figura 3 - Resposta da variável de saída $\left(\mathrm{h}_{2}\right)$ frente a uma perturbação degrau na vazão de entrada $\left(\mathrm{q}_{1}\right)$.

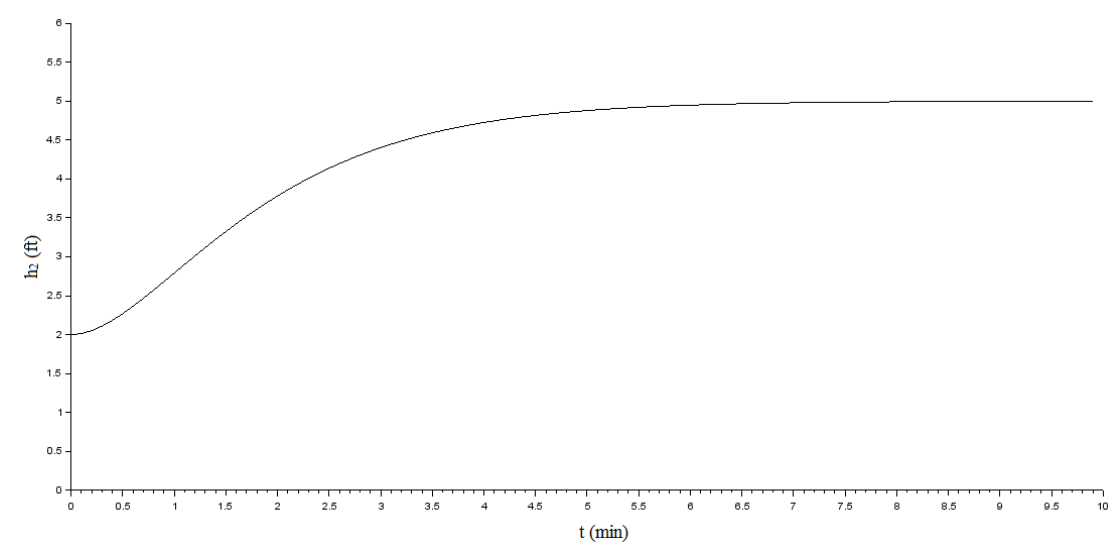

Figura 4 - Diagrama de controle implementado no xcos.

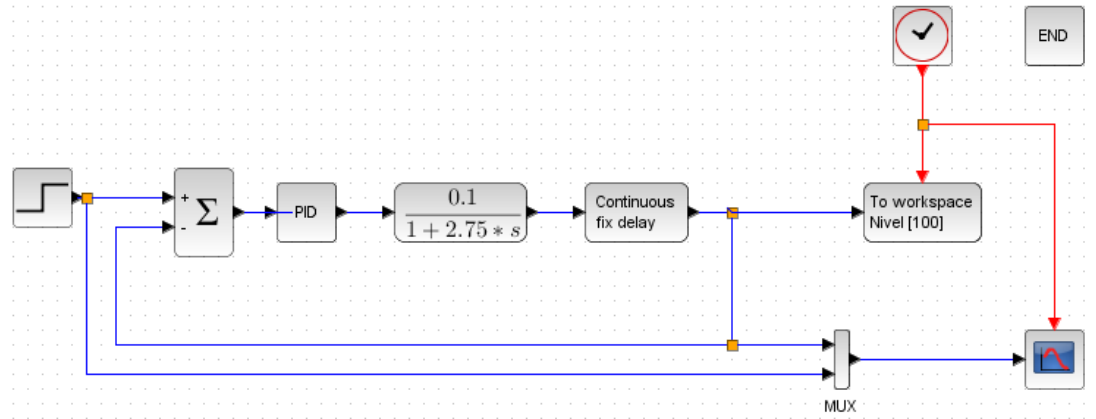

O bloco "Continuous fix delay" é utilizado para aplicar o tempo morto da aproximação para função transferência do processo de primeira ordem. Uma perturbação degrau é configurada com o valor da variação do setpoint escolhido, que é comparado com a resposta do sensor, obtendo um erro o qual se espera diminuir com o controlador. Uma linha de referência evidencia o valor do setpoint, a fim de fornecer uma perspectiva visual do offset.

\subsection{Sintonia do Controlador PID}

Utilizando o método "Ultimate Gain" a partir de uma perturbação degrau do setpoint de $1 \mathrm{ft}$, elevou-se gradativamente o ganho proporcional até um valor crítico $\mathrm{K}_{\mathrm{Cr}}$ em que ocorre uma oscilação sustentada (limite da estabilidade) na variável de saída, a fim de se determinar o período das oscilações $\mathrm{T}_{\mathrm{c}}$, o gráfico gerado pelo software no ganho crítico $\mathrm{K}_{\mathrm{Cr}}$ de 179 é apresentado na Figura 5. O período das oscilações $\mathrm{T}_{\mathrm{c}}$ é aferido de aproximadamente 1,0 min.

Com os parâmetros $\mathrm{K}_{\mathrm{Cr}}$ e $\mathrm{T}_{\mathrm{c}}$ pode-se determinar o ganho proporcional $\left(\mathrm{K}_{\mathrm{C}}\right)$, tempo integral $\left(\tau_{\mathrm{I}}\right)$ e tempo derivativo $\left(\tau_{\mathrm{D}}\right)$ do controlador PID, pelos métodos de Ziegler-Nichols e Tyreus-Luyben, conforme a Tabela 1. Os parâmetros calculados são dispostos na Tabela 2.

A Figura 6, apresenta os gráficos resultantes das sintonias segundo Ziegler-Nichols e Tyreus-Luyben, para uma perturbação degrau no setpoint de $5 \mathrm{ft}$. Percebe-se que a primeira 
sintonia (esquerda) apresentou maior oscilação em relação a segunda (direita), porém a segunda sintonia apresentou um maior offset ao longo do tempo.

Figura 5 - Oscilação sustentada do nível $\left(\mathrm{h}_{2}\right)$.

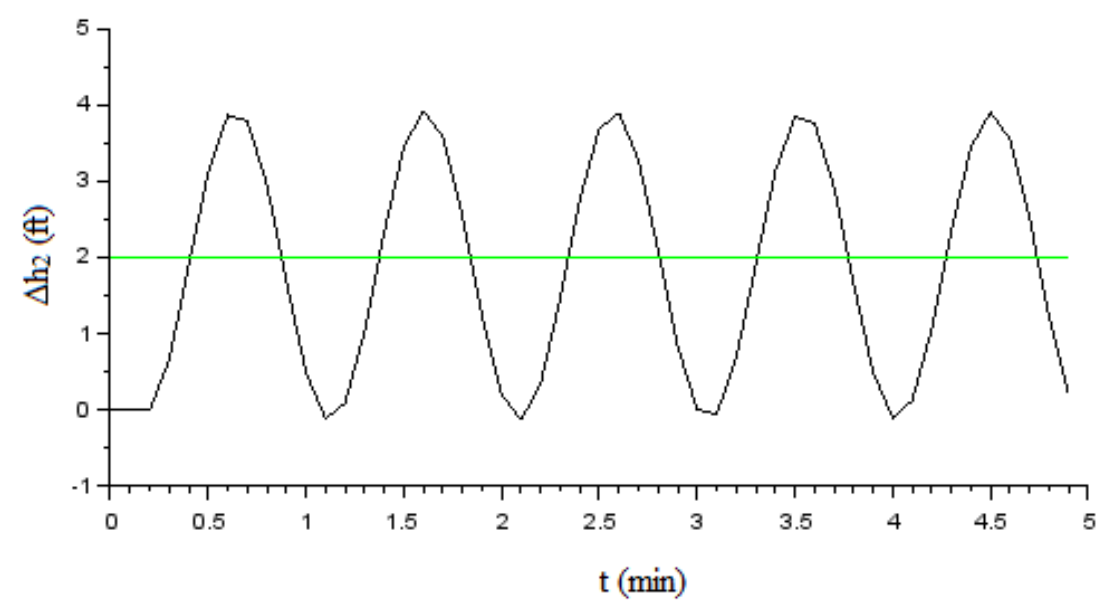

Tabela 1 - Métodos de Sintonia PID

\begin{tabular}{cccc}
\hline Ziegler-Nichols & $0,6 \mathrm{Kcr}$ & $0,5 \mathrm{Tc}$ & $0,125 \mathrm{Tc}$ \\
Tyreus-Luyben & $0,45 \mathrm{Kcr}$ & $2,2 \mathrm{Tc}$ & $0,158 \mathrm{Tc}$ \\
\hline
\end{tabular}

Tabela 2 - Sintonia do Controlador

\begin{tabular}{lccc}
\hline Ziegler-Nichols & 107,000 & 0,5 & 0,125 \\
Tyreus-Luyben & 80,600 & 2,200 & 0,158 \\
\hline
\end{tabular}

Figura 6 - Sintonias de Ziegler-Nichols (esq) e Tyreus-Luyben (dir)
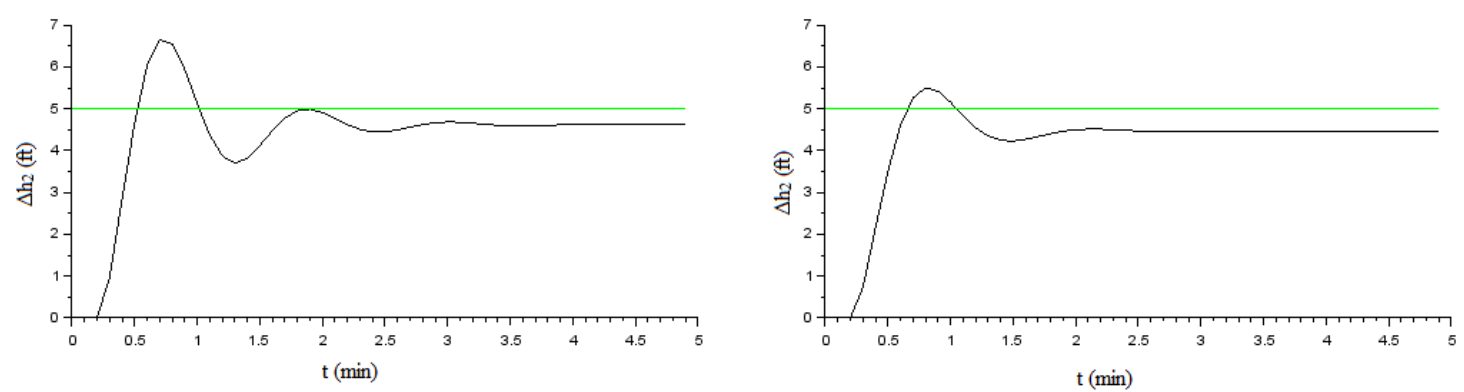

Para utilização do critério de integração do erro, ponderado no tempo, dado pela equação 3, foi implementado no diagrama de bloco e verificado os valores obtidos pelos métodos Ziegler-Nichols e Tyreus-Luyben e obteve-se um ITAE menor para o método de Ziegler-Nichols. Assim, partiu-se dos valores obtidos do método de sintonia de ZieglerNichols e encontrou-se valores que minimizam o critério, sendo selecionados para utilização 
do controlador PID, apresentando uma diminuição nas oscilações e offset, cujos valores são: $\mathrm{K}_{\mathrm{C}}=100 ; \tau_{\mathrm{I}}=0,05 ; \tau_{\mathrm{D}}=10,0$; Graficamente obteve-se a resposta conforme a Figura 7.

\section{CONCLUSÕES}

Este trabalho possibilitou implementar e sintonizar um controlador PID em um sistema de tanques em série. Para isso, foi aplicado o método "Ultimate Gain", aplicando perturbação no sistema até a saída exibir uma oscilação sustentada (amplitude constante). Porém, esse método necessita de um tempo de sintonia alto, uma vez que necessita de diversas tentativas, como também, submeter o processo no limite da estabilidade em situações reais, pode acarretar em operação instável e resultar em situação de perigo. Sendo assim, sugere-se utilizar o método de Astrom e Hanglund, na qual o sistema é forçado através de um controlador on-off ou um relé, a oscilar com pequenas amplitudes.

Concluiu-se também, que a ferramenta $x c o s$ do software gratuito Scilab se mostrou de grande utilidade para o estudo e implementação do controlador PID.

Figura 7 - Ajuste dos parâmetros do PID baseado no ITAE.

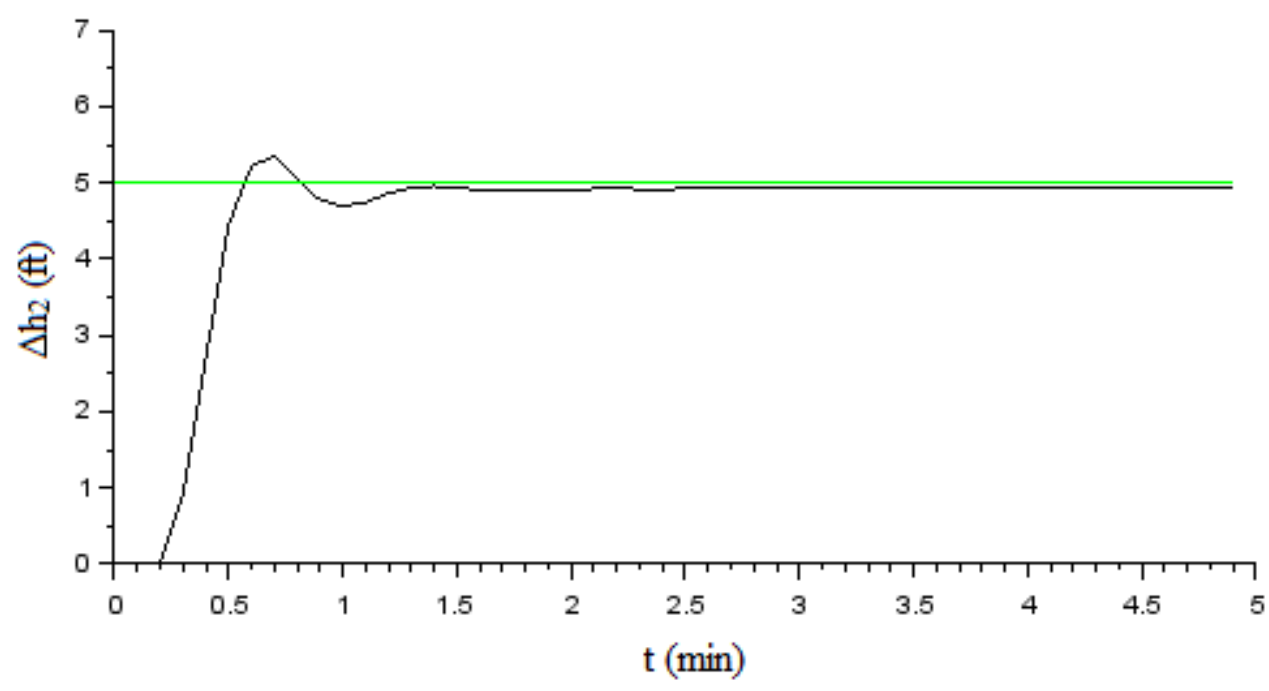

\section{REFERÊNCIAS}

FRANCHI, C. M. Controle de Processos Industriais. 1. ed. São Paulo: Érica, 2011.

HAUGEN, Finn. Comparing PI tuning methods in a real benchmark temperature control system. Modeling, Identification and control, v. 31, n. 3, p. 79, 2010.

JABUONSKI JR, R. E. et al. Sistema de experimentação remota configurável de controle de nível de líquidos multivariável. VI SBAI, Buaru, Brasil, 2003.

MARTINS, Fernando G. Tuning PID controllers using the ITAE criterion. International Journal of Engineering Education, v. 21, n. 5, p. 867, 2005. 